\title{
GA-BASED DYNAMIC ROUTING IN WDM OPTICAL NETWORKS
}

\author{
G. Ramesh ${ }^{1}$ and S. Sundara Vadivelu ${ }^{2}$ \\ ${ }^{1}$ Research Scholar, Anna University, Chennai, ${ }^{2}$ Professor/ECE, SSN College of Engineering, Chennai \\ ramesh6_2000@yahoo.com, sundaravadivelus@hotmail.com
}

\begin{abstract}
The enormous increase in online content and the requirement of providing universal access to broadband Internet services is already stretching the capabilities of current technologies to their limit. New applications require networks with well-known and predictable characteristics and behavior. Advances in optical networks, together with the rapid rising demand for network bandwidth, are fueling an increasing amount of research in the field of optical networks. Optical communication has become a promising networking choice to meet increasing demand on bandwidth. Some of the emerging bandwidth-intensive computing and communication applications such as data browsing on the web, video conferencing, e-commerce, high-definition video/audio on-demand processing, data mining, database and decision-support transactions etc. The objective of this paper is to minimize the number of wavelengths, converters or other cost parameters. For this the network path should be an optimized one. This problem can be formulated as a mixed integer linear program, which is NP-complete. Performance has been evaluated with three different networks, a small, medium and large sized network with 6-node, 10-node and 14-node networks, respectively. Analysis shows that the Genetic Algorithm (GA) based method provides an optimized route with significantly less blocking probability than the normal cases.
\end{abstract}

Keywords: Light path, genetic algorithms, wavelength division multiplexing, optical network, routing.

\section{INTRODUCTION}

With fast growth of the Internet and World Wide Web, the network bandwidth requirements have increased dramatically in recent years. The research and technology development in Wavelength Division Multiplexing (WDM) networks are now evolving at a staggering pace to fulfill the increasing bandwidth requirement and the deployment of new network services [3]. All optical networking based on Wavelength Division Multiplexing (WDM) has attracted considerable interest in recent years. Rapid growth of bandwidth requirements and excess bandwidth available in optical fibers are the major driving forces of this attention. In WDM networks, vast bandwidth in the range of $25-50 \mathrm{THz}$ are available in optical fibers is accessed in smaller manageable channels each on a different wavelength. There are many ways to organize a WDM all-optical network [19].

The main objective of the optical network is to increase the acceptance ratio or to decrease the blocking probability of the connections in the network. For this achievement heuristic algorithms are be used. To solve this problem three different methods are used namely: fixed, fixed-alternative and dynamic approach [17]. To establish a lightpath a set of pre-computed shortest paths are used in the fixed and fixed-alternative approach. These two approaches are very simple and have less setup time and also low control overhead. But these approaches results in high blocking probability.

The dynamic approach is more efficient than the fixed and fixed-alternative approaches. In dynamic approach the blocking probability is much better than the other two. This is because the route is chosen based on the current state of the network. But the dynamic approach requires a long setup time and a high control overhead. One of the key benefits of optically switched networks is the transparency to bit-rate, protocol or modulation format [12]. This is also one of the main obstacles when determining an optimum path through the network. Intelligent optical network management systems allow carries to set up new routes through the network in a matter of seconds or minutes, rather than the weeks or months it takes with legacy systems.

The remaining paper is organized as follows. Section 2 describes the review of literature on wavelength division multiplexing networks. Section 3 describes about the routing framework for wavelength-routed WDM networks with the genetic algorithm. The experimental scenario arrangements are detailed in section 4 . Section 5 illustrates with the results and its analysis. Finally a conclusion is added in section 6.

\section{RELATED WORK}

Michael et al [13] have presented an L+1 fault tolerance which is used for the recovery of optical networks from single link failures without the allocation of valuable system resources. While the approach in its simplest form performs well against the existing schemes, the flexibility of $\mathrm{L}+1$ leave many options to examine possible ways to further increase performance. Muriel M'edard [15] has described that the protection routes are pre-computed at a single location and thus it is centralized. Before the restoration of the traffic, some distributed 
reconfiguration of optical switches is essential. On the other hand, restoration techniques depend upon distributed signaling between nodes or on the allocation of a new path by a central manager.

Hongsik et al [7] have considered the network survivability which is a critical requirement in the high-speed optical networks. A failure model is considered so that any two links in the network may fail in a random order. They have presented three loop back methods of recovering from double-link failures. Only the first two methods require the identification of the failed links. But pre-computing the backup paths for the third method is more complex than the first two methods. The double link failures are tolerated by the heuristic algorithm which pre-computes the backup paths for links.

Yufeng et al [24] have studied the important fault management issue which concentrates on the fast restoration mechanisms for Optical Burst Switched (OBS) networks. The OBS network operates under the JIT signaling protocol. The basic routing mechanism is similar to the IP networks, where every OBS node maintains a local forwarding table. The entries in the forwarding table consist of the next hop information for the bursts per destination and per FEC (Forward Equivalent Class). Based on looking up the next-hop information in their forwarding tables, OBS nodes forwards the coming burst control packets and set up the connections. The connection set up process is signified by the burst forwarding or burst routing.

Jian et al [10] have considered the fault-monitoring functions which are usually provided by the optical-transmission systems. In order to measure the bit error rate in the wavelength channels using SONET framing, the B1 bit in the SONET overhead can be used. Moreover, to detect certain failures like fiber cut in other formatted optical channels, the optical power loss can be used. Optical-Electrical-Optical (OEO) conversion is used before each OXC port because most of the OXCs use electronic switching fabric. Therefore, faults can be detected on link-bylink basis. Both the end nodes of the failed link can detect the fiber cut for all-optical switches.

Lei Guo [11] has studied the problem of multiple failures in WDM networks. In order to improve the survivable performance he proposed a heuristic algorithm called Shared Multi-sub backup paths Reprovisioning (SMR). The survivable performance of SMR in multiple failures was considerably improved when compared with the previous algorithm.

Guido et al [6] have investigated the issue of dynamic connections in WDM networks. It is also loaded with the highpriority protected static connections. They have compared various routing strategies by discrete event simulation in terms of blocking probability. Based on the occupancy cost function they have proposed a heuristic algorithm which takes several possible causes of blocking into account. The behavior of their algorithm was tested in well known case study of mesh networks, with and without wavelength conversion.

Rajkumar et al [16] have proposed a distributed priority based routing algorithm. In order to establish the primary and backup light paths they have proposed a variety of traffic classes which uses the concept of load balancing. Based on the load on the links, their algorithm estimates the cost metric. The routing of high priority traffic was performed over the lightly loaded links. Therefore while routing the primary and backup paths, the lightly loaded links are chosen instead of choosing the links with heavier loads. The load balancing will not reflect the dynamic load changes because it is used in the routing metric.

Dong et al [4] have developed two heuristic multipath routing schemes for survivable multipath problem called CPMR (Conditional Penalization Multipath Routing) and SPMR (Successive Penalization Multipath Routing). Their schemes use "link penalization" methods to control (but not prohibit) linksharing to deal with the difficulties caused by the link sharing. When compared with the routing scheme that searches for disjoint paths, their methods have considerably higher routing success rates which are shown through the simulation results.

Ramamurthy et al [18] showed that the presence of wavelength converters significantly improves the performance in networks that employ alternate routing when compared to networks that use fixed routing. They also showed that the benefits of adding an alternate route is more than the benefits of adding a wavelength converter at low loads when the number of alternate routes between node pairs does not fully exploit the connectivity of the network topology.

Der-Rong [2] proposed the WDM Virtual Topology Configuration Transition Problem (WVTCTP) which minimizes the average weighted hop distance is studied. Since the WVTCTP is NP-hard, a Genetic Algorithm (GA) is proposed to solve it. Simulated results show that the proposed GA can get better performance than heuristic, simulated annealing, and iterative improving methods.

Ignacio et al [9] proposed a novel genetic algorithm to solve the joint routing and dimensioning problem in dynamic WDM networks, with the aim of obtaining a network cost close to minimum while guaranteeing an upper bound on the blocking probability. Used prior to network operation, the algorithm determines which route should be used for each potential connection and also dimensions the number of wavelengths required in each link. The efficiency of the algorithm is validated in ring and mesh topologies, providing wavelength savings of up to $17 \%$ when compared with the best existing algorithm to date. Moreover, since the routes provided by the genetic algorithm are stored in routing tables, it also ensures extremely fast on-line network operation. 
Mohan et al [14] proposed two different algorithms named the Primary Dependent Backup Wavelength Assignment (PDBWA) and the Primary Independent backup Wavelength Assignment (PIBWA) for the problem of dynamic survivable routing in WDM optical networks. The proposed algorithms uses a backup multiplexing technique, which allows backup lightpaths to use the same wavelength on the same link if the primary lightpaths are disjoint links.

Sen et al [20] proposed to use the link-disjoint path pair, whose longer path is shortest among all such pairs of paths, for path protection so that the delay on the backup path is minimized. They prove that the problem of finding such a pair of paths is NP-complete, and they use the one-step approach as the approximation solution. Xin et al [23] attempted to optimize the network resource utilization of each call by minimizing the overall cost of the primary and backup path. The paths are selected from $\mathrm{K}$ precomputed candidate route pairs.

Srinivasan [21] developed a framework to support multiple protection strategies in optical networks, which is in general applicable to any connection-oriented network. The capacity available on a link for routing primary and backup connections is computed depending on the protection strategy. They also developed a model for computing service outage and failure recovery times for a connection where notifications of failure location are broadcast in the network. The effectiveness of employing multiple protection strategies is established by studying the performance of three networks for traffic with four types of protection requirement.

\section{GENETIC ALGORITHM}

Genetic Algorithms are search algorithms based on biological evolution models. The algorithm operates on a population of individuals which represent possible routes in the search space. The algorithm begins with an initial population of individuals; each of them represents a feasible solution to the problem being tackled. Each route has some fitness value and is measured by an evaluation function. The approach of the algorithm is to explore the search space and to discover a better optimal route by permitting the individuals to evolve over time [22].

Figure 1 shows the flow chart of the genetic algorithm as a function of multicast routing. Each reproduction creates a new generation, starting from an existing generation and strings are reproduced with a probability, which is proportional to the quality of the corresponding solution. In each generation, strings that represent solutions with good properties have a higher chance to survive than the others [1].

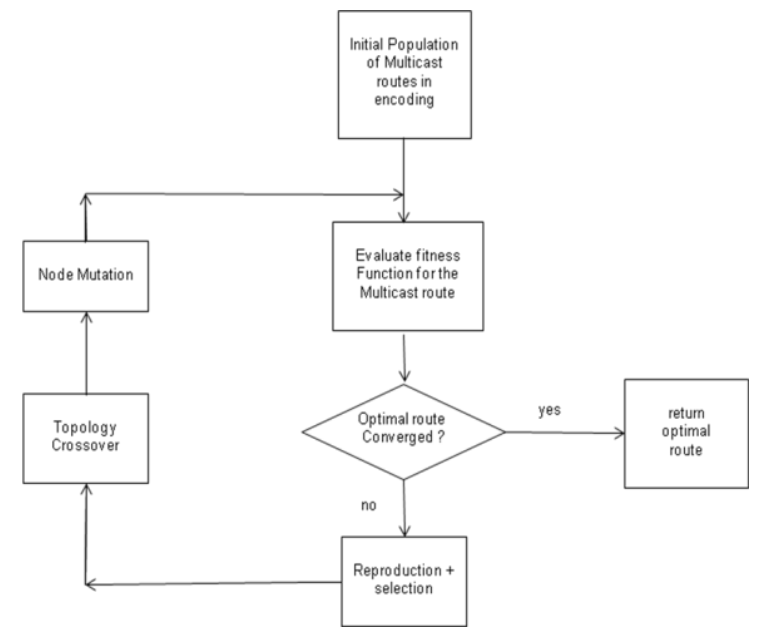

Figure 1 Genetic Algorithm flowchart

The crossover operator chooses a pair of strings, which breaks up their gene sequence at random places, and exchanges the genetic information. The mutation operator introduces new genetic material by randomly selecting and changing simple genes. The result of crossover and mutation will generate a better population and the process is repeated until a good solution is found or until a predefined number of iteration [5].

The major advantage of the GA approach is that it does not rely upon specific knowledge of the problem definition. The success of the algorithm is attributed to various factors, including its powerful parallel search capability, computation simplicity, robustness, global search capability, ability to combine with other heuristic procedures and independence from solution characteristics such as linear or non-linear constraints and discrete or continuous search space.

\section{EXPERIMENTAL SETUP}

A network consists of nodes, and links connecting the nodes. This can be modeled by a graph, with a node as a vertex and a link as an edge. In this section, the performance of the proposed algorithms is evaluated in a small, medium and large sized network with 6-node, 10-node (Abilene) and 14-node National Science Foundation NETwork (NSFNET), which is shown in figure 2, 3 and 4 respectively.

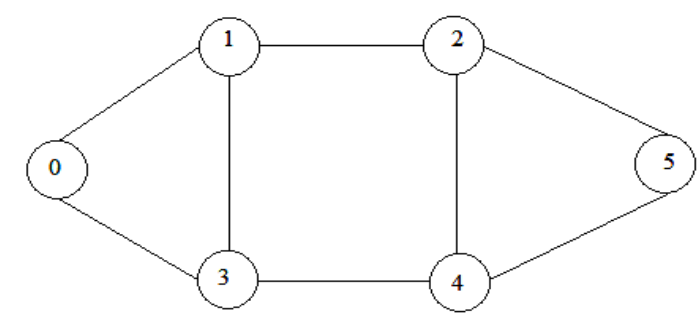

Fig. 2 A 6-node network 


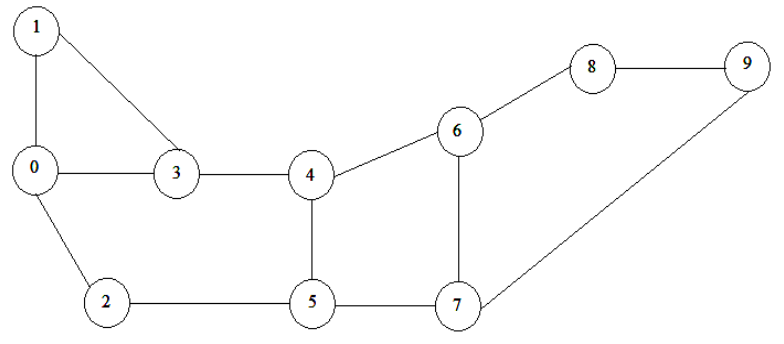

Fig. 3 A 10-node (Abilene) network

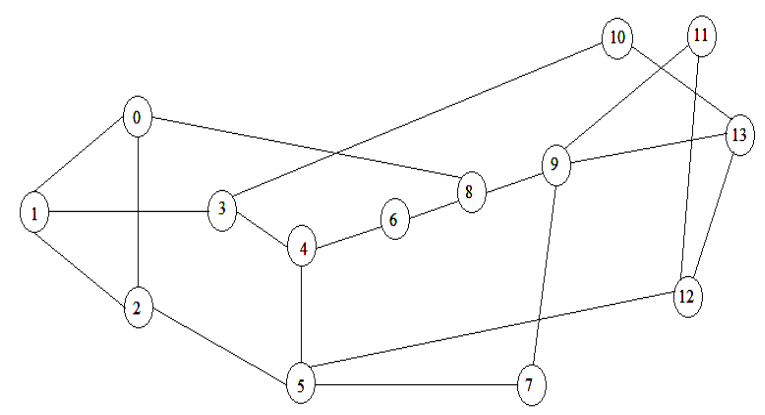

Fig. 4 A 14-node NSFNET

To apply the GA, the mesh structure is transformed into a spanning tree structure using Kruskal's algorithm. The nodes in the network form a tree, and the number of distinct spanning trees on a complete graph of ' $\mathrm{n}$ ' nodes is $\mathrm{nn}-2$ according to Prufer's proof of Cayley's theorem. Then an initial population is generated randomly. This population is then evolved by genetic operators; the crossover and mutation operators. For this one point crossover and node mutation is used. After applying the genetic operators the reproduction stage selects the fittest individuals that have the highest fitness value from parents and children, for the next generation. This process is repeated until the stopping condition is fulfilled and the best individual is selected.

These topologies were selected as representative topologies to understand how routing performance might be impacted differently on different topologies. All links in these networks were assumed to be bidirectional multi-wavelength fibers with the same number of wavelengths in each fiber. The setup is assigned that at each network with no wavelength converters and determined a baseline load such that the call blocking probability is approximately $1 \%$ with two routes in the routing table for all traffic cases.

\section{SIMULATION RESULTS}

The performance of the proposed algorithm was examined with an extensive simulation study based upon the NS 2 network simulator [8]. For the simulation a dynamic traffic model was used in which connection requests arrive to a node according to the Poisson process. The arrival rate of the packet to a node in the Poisson process can be given by $\square$ call/seconds. The destination node was selected randomly from all the other nodes except the node which is selected as source.

The connection holding time for the above process was exponentially distributed with a mean holding time of $1 / \mu$ seconds. The connection request was distributed randomly on all the nodes in the network. For the entire process the request requires a full wavelength bandwidth capacity. Figure 5, 6 and 7 illustrates the analysis of average tree cost vs required computing resources, blocking probability vs routes in routing table and blocking probability vs loads for three different networks (6 node, 10 node and 14 node) respectively. From the analysis, it is observed that when the number of nodes increases in the network, then the corresponding tree cost or the blocking probability also get increased. The tree cost seems to be linear when there is a uniform increase in the computing resources. As GA is considered, it will not give good performance initially, but once the tree is constructed which is an optimized one, then the results will be better.

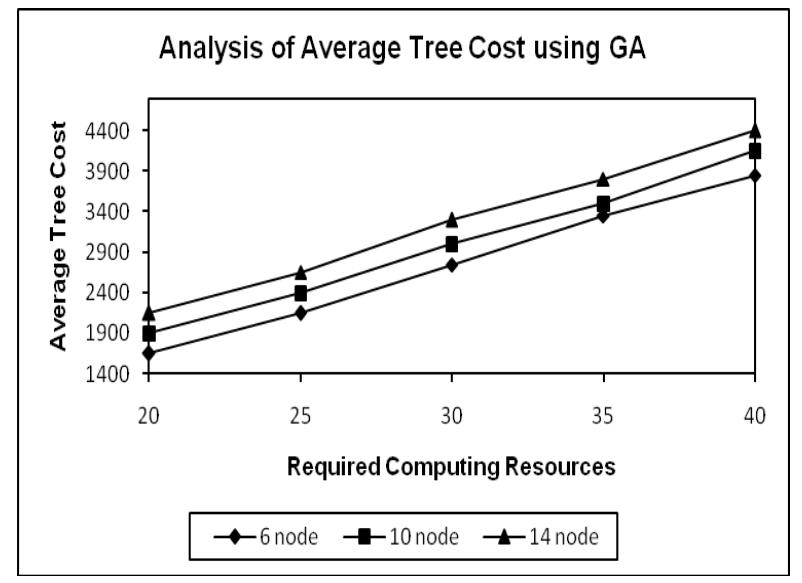

Fig. 5 Analysis of average tree cost using GA

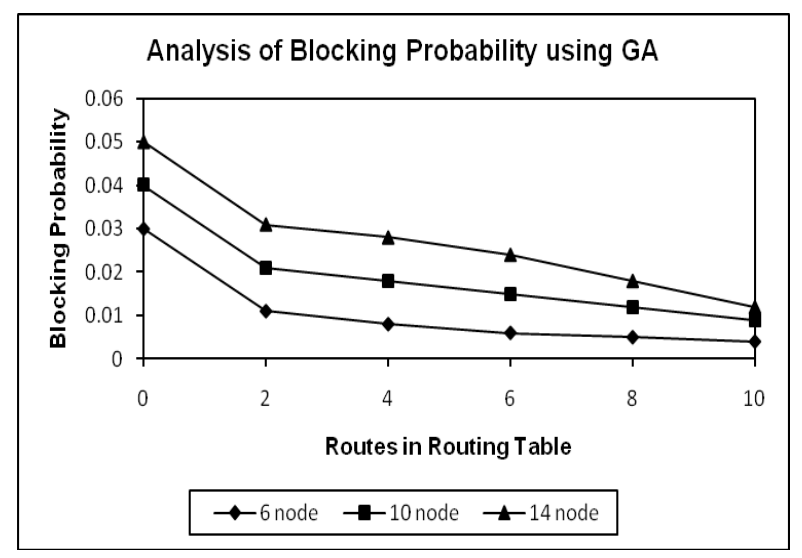

Fig. 6 Analysis of blocking probability using GA 


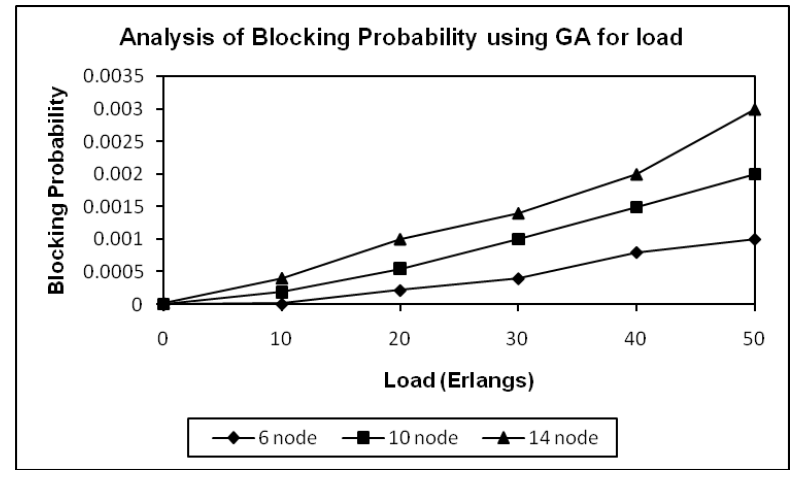

Fig. 7 Analysis of blocking probability using GA for load

\section{CONCLUSIONS}

This paper discussed a genetic algorithm based optimized routing strategy in wavelength division multiplexing optical networks. The proposed approach shows that the blocking probability is improved. Future optical networks, especially the metropolitan networks and local networks are expected to flexibly and cost effectively satisfies a wide range of applications having time-varying and high bandwidth demands and stringent delay requirements. Another characteristic of a future proof network, such as reliability, survivability and coverage, can be improved by a network topology that is scalable and enables traffic routing.

\section{REFERENCES}

[1]. Bisbal D., et al., "Dynamic routing and wavelength assignment in optical networks by means of genetic algorithms", Photonic Network Communications, 7 (1), pp. 43-58, 2004.

[2]. Der-Rong Din, "A genetic algorithm for solving virtual topology configuration transition problem in WDM network", International Journal of Computer Communications, Volume 30, Issue 4, Pages 767-781, 26 February doi:10.1016/j.comcom.2006.09.009.

[3]. Deying Li, Xiaohua Jia, Xiao-Dong $\mathrm{Hu}$ and Eric Z. Gong, "Wavelength assignment for minimizing system blockings in multifiber all-optical WDM networks", Optical Networks Magazine, pp. 75-82, July/August 2003.

[4]. Dong-won Shin, Edwin K. P. Chong and Howard Jay Siegel, "Survivable Multipath Routing Using Link Penalization", Computing and Communications, 2004 IEEE International Conference.

[5]. Goldberg D. E., "Genetic Algorithms in Search, Optimization, and Machine Learning", Addison-Wesley Publishing Company Inc., 1997.

[6]. Guido Maier, Achille Pattavina, Luigi Barbato, Francesca Cecini and Mario Martinelli, "Routing Algorithms in WDM Networks under Mixed Static and Dynamic Lambda-Traffic", Journal on Photonic Network
Communications, Vol. 8, No. 1, pp. 69- 87, June 2004, Doi: 10.1023/B:PNET.0000031619.18955.b4.

[7]. Hongsik Choi, Suresh Subramaniam, and Hyeong-Ah Choi, "On Double-Link Failure Recovery in WDM Optical Networks", INFOCOM, Volume 2, pp. 808- 816, 2002.

[8]. http://www.isi.edu/nam/ns/index.html, 2003. Ignacio de Miguel, Reinaldo Vallejos, Alejandra Beghelli, and Ramon J. Duran, "Genetic Algorithm for Joint Routing and Dimensioning of Dynamic WDM Network", Journal of Optical Communications and Networking, Vol. 1, Issue 7, pp. 608-621, 2009, doi:10.1364/JOCN.1.0 00608.

[9]. Jian Wang, Laxman Sahasrabuddhe and Biswanath Mukherjee, "Fault Monitoring and Restoration in Optical WDM Networks", National Fiber Optic Engineers Conference, NFOEC, 2002.

[10]. Lei Guo, "Heuristic Survivable Routing Algorithm for Multiple Failures in WDM Networks", in Proceedings of $2^{\text {nd }}$ IEEE/IFIP International Workshop on Broadband Convergence Networks, pp. 1-5, 21 May 2007, Doi: 10.1109/BCN.2007.372750.

[11]. Marsden A., Maruta A., and Kitayama K., "Dynamic RWA with FWM-induced crosstalk awareness and modulation format conversion in wavelength-routed optical networks", Journal of Optical Switching and Networking, 6, pp. 79-87, 2009.

[12]. Michael T. Frederick and Arun K. Somani, "A singlefault recovery strategy for optical Networks using sub graph routing", The International Journal of Computer and Telecommunications Networking, Volume 50, Issue 2, February 2006.

[13]. Mohan G., Murthy C. S. R., and Somani A. K., "Efficient algorithms for routing dependable connections in WDM optical networks, IEEE Transactions on Networking 9 (5), pp. 553-566, 2001.

[14]. Muriel M'edard, "Network Reliability and Fault Tolerance", IEEE Transactions on Volume 50, Issue 1, pp. 85 - 91, Mar 2001.

[15]. Rajkumar A. and Murthy Sharma N. S., "A Distributed Priority Based Routing Algorithm for Dynamic Traffic in Survivable WDM Networks", International Journal of Computer Science and Network Security, Vol.8, No.11, November 2008.

[16]. Ramamurthy and Mukherjee B., "Fixed-alternate routing and wavelength conversion in wavelength-routed optical networks", IEEE/ACM Transaction on Networking, 10, (3), pp. 351-367, 2002.

[17]. Ramamurthy R., Sahasrabuddhe L., and Mukherjee B., "Survivable WDM mesh networks", Journal of Lightwave Technology, 21 (4), pp. 870-883, 2003.

[18]. Ramaswami R., "Multi-wavelength Light wave Networks for Computer Communication", IEEE Communications Magazine, pp. 78-88, February 1993.

[19]. Sen A., Shen B. H. and Bandyopadhyay S., "Survivability of lightwave networks-path lengths in 
WDM protection scheme", Journal of High Speed Networks, Vol. 10, pp. 303-315, 2001.

[20]. Srinivasan Ramasubramanian, "Supporting Multiple Protection Strategies in Optical Networks", IEEE/ACM Transaction on Networking, Vol. 16, No. 6, pp. 1352-1365, December 2008.

[21]. Talay A. C., and Oktug S., "A GA/Heuristic based Hybrid Technique for Routing and Wavelength Assignment in WDM Networks", Proceedings of the EvoComNet'04, Coimbra, Portugal, in LNCS, Vol. 3005, pp. 150-159, 2004.

[22]. Xin C., Ye Y., Dixit S. and Qiao C., "A joint lightpath routing approach in survivable optical networks", Proceedings of the SPIE Asia-Pacific Optical and Wireless Communications, Vol. 4585, pp. 139-146, Nov. 2001.

[23]. Yufeng Xin, Jing Teng, Gigi Karmous-Edwards, George N. Rouskas and Daniel Stevenson, "Fault Management with Fast Restoration for Optical Burst Switched Networks", IEEE, 2004.

\section{BIOGRAPHIES}

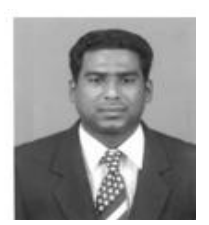

G. Ramesh is working as Assistant Professor in the Department of Information Technology at KLN College of Engineering, Madurai, Tamil Nadu, India. $\mathrm{He}$ has completed graduation in Electrical and Electronics Engineering and Post Graduation in Computer Science and Engineering. He is doing research work in optical networking.

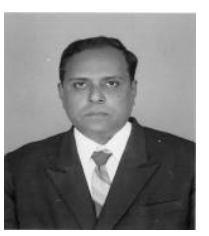

S. Sundara Vadivelu is working as Professor in Department of Electronics and Communication Engineering at SSN College of Engineering, Kalavakkam, Chennai, Tamil Nadu India. His research interest is in Optical Communication and Networks. He has published many international and national journals in optical domain. 